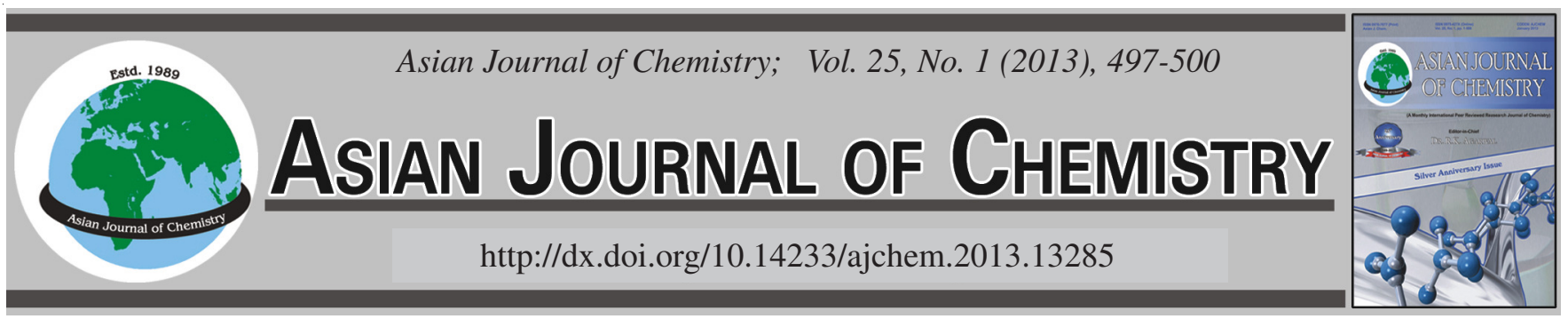

\title{
Synthesis of Ordered Mesoporous Carbons and Adsorption Equilibrium of Resorcinol
}

\author{
Chen Ren-Hong ${ }^{1, *}$, YuAn Ping and Wang XiU-FAng ${ }^{2}$
}

${ }^{1}$ Guangdong Food and Drug Vocational College, LongDong North Road, TianHe Restrict, Guangzhou 510520, P.R. China

${ }^{2}$ College of Pharmacy, Guangdong Pharmaceutical University, Guangzhou Higher Education Mega Center, Guangzhou 510006, P.R. China

*Corresponding author: Tel/Fax: +86 20 37214322; E-mail: chenrenhong306@163.com

(Received: 19 December 2011;

Accepted: 2 August 2012)

AJC-11909

\begin{abstract}
Ordered mesoporous carbons were synthesized by using SBA-15 as template, furfuryl alcohol as carbon precursor and oxalic acid as catalyst. The influence of the usage of oxalic acid on the pore structure of the resulting materials was evaluated. Characterizations such as X-ray diffraction, nitrogen sorption, transmission electron microscopy and Raman spectroscopy were carried out. The adsorption behaviour of resorcinol on the obtained ordered mesoporous carbons was investigated. With the increasing usage of oxalic acid, the structural order, unit cell parameter and wall thickness tended to increase but followed by a small decrease. The BET specific surface areas and pore volumes showed decreasing tendency and pore diameters had the smallest value when using $0.1 \mathrm{~g}$ of oxalic acid. The adsorption of resorcinol onto ordered mesoporous carbons followed the Langmuir-Freundlich equation.
\end{abstract}

Key Words: Ordered mesoporous carbons, Synthesis, Adsorption, Resorcinol.

\section{INTRODUCTION}

Ordered mesoporous carbon (OMC), as a new functional material $^{1-6}$ has attracted great technological interest for the development of adsorption and purification of water, electronic, catalytic and energy storage systems, due to their remarkable properties such as high specific surface area, narrow pore size distribution, large adsorption capacity and high thermal stability. It has great application value in advanced material design, drug release and adsorption ${ }^{7-10}$. Phenols are the most prevalent organic pollutants in wastewaters and impart disagreeable tastes and odours to drinking water, taint fish flesh and exert various other adverse environmental effects at very low concentrations ${ }^{11}$. So, it is necessary to remove the phenolic impurities from wastewater. Adsorption could be used as an effective technique in the treatment of wastewaters ${ }^{12,13}$. However, the adsorption of resorcinol on ordered mesoporous carbons was not covered in the literature.

Herein, a series of ordered mesoporous carbons were synthesized by using the oxalic acid as the catalyst. The effect of the amount of the oxalic acid catalyst on the final synthesis of ordered mesoporous carbon structure was investigated. By use of resorcinol as a model pollutant, the adsorption of resorcinol on ordered mesoporous carbons was studied and the adsorption data were fitted by Langmuir-Freundlich equation and parameters were obtained. The advantage of this precess was that there were no metal ions or halogen ions introduced during the experiment process, providing a base for the further study of ordered mesoporous carbon in adsorption and separation.

\section{EXPERIMENTAL}

SBA-15-100 was synthesized by using the hard template method described by Zhao et al. ${ }^{2}$. Ordered mesoporous carbon was synthesized using the furfuryl alcohol as the carbon source and the oxalic acid as the catalyst. The typical experimental process was as follows: $1 \mathrm{~g}$ of SBA-15-100 was impregnated in $2 \mathrm{~mL}$ of furfuryl alcohol/TMB solution with the volume ratio of $4: 1$ by the incipient wetness impregnation. Then 0.03 , $0.1,0.2 \mathrm{~g}$ of oxalic acid were added, respectively, as a polymerization catalyst. The mixture thus prepared was heated at $80^{\circ} \mathrm{C}$ for $12 \mathrm{~h}$ under vacuum for the polymerization of furfuryl alcohol and then at $150{ }^{\circ} \mathrm{C}$ for $6 \mathrm{~h}$. After cooling to room temperature, the sample was heated to $300^{\circ} \mathrm{C}\left(1{ }^{\circ} \mathrm{C} / \mathrm{min}\right)$, then to $850^{\circ} \mathrm{C}\left(5^{\circ} \mathrm{C} / \mathrm{min}\right)$ for $4 \mathrm{~h}$ under flowing $\mathrm{N}_{2}$ atmosphere in a tubular furnace (KTL-1400). The obtained black powder was washed by $20 \%$ of $\mathrm{HF}$, then with water to neutral, finally dried under vacuum at $40{ }^{\circ} \mathrm{C}$ for $24 \mathrm{~h}$. The sample obtained was marked as OMC-X, where $\mathrm{X}$ referred the amount of oxalic acid. The water used in this experiment was the ultra-pure water and all reagents were of analytical grade.

Detection method: The samples were characterized by powder X-ray diffraction (XRD, PANanalytical. Inc. X'Pert Pro., MPD), nitrogen adsorption analyzer (Micromeritics 
Tristar 3020), transmission electron microscopy (TEM, EOL 2011 microscope operated at $200 \mathrm{kV}$ ) and Raman spectroscopy (HORIBA Jobin Yon LabRAM, He-Ne emitter, 633nm). Before nitrogen absorption test, the sample was first degassed under a vacuum at $200^{\circ} \mathrm{C}$ for $4 \mathrm{~h}$. The specific surface area was calculated by BET method. The pore size distribution was obtained by fitting the data of absorption isotherm using BJH model. The pore volume was calculated from the cumulative absorption volume at a relative pressure of 0.99 .

Adsorption of resorcinol: $5 \mathrm{mg}$ ordered mesoporous carbons were added into $25 \mathrm{~mL}$ of resorcinol solution with different concentrations. The resulting mixture was continuously stirred in a closed batch at $25^{\circ} \mathrm{C}$ until equilibrium. This was done by monitoring the resorcinol concentration using a UV-vis spectrophotometer at a wavelength of $270 \mathrm{~nm}$. The adsorbed amount of resorcinol was determined according to the change of concentration before and after adsorption.

\section{RESULTS AND DISCUSSION}

Fig. 1 showed the XRD patterns of mesoporous carbons synthesized with different amount of oxalic catalyst. The synthesized mesoporous carbons had clear 100, 110, 200 diffraction peaks, indicating the hexagonal structure with p6mm space group and the well-ordered structure. The corresponding $\mathrm{d}_{100}$-spacing value was calculated as $9.86 \mathrm{~nm}$. The lattice parameter $\alpha$ and the wall thickness were listed in Table-1. In the range of this study, with the increase of the amount of oxalic acid $(0.03,0.1,0.2 \mathrm{~g})$, the Bragg angles $2 \theta$ corresponding to the peak 100 were $0.99^{\circ}, 0.96^{\circ}, 0.99^{\circ}$ respectively, indicating that the structural order increased at first and then decreased with the increase of the amount of oxalic acid catalyst and it was relatively better when the amount of the catalyst was $0.1 \mathrm{~g}$. The same trend can also be seen from Fig. 1 , that was, the lattice parameter $\alpha$, the wall thickness and $d_{100}$ value all increased at first and then decreased with the increase of the amount of oxalic acid catalyst, they were relatively larger when the amount of the catalyst was $0.1 \mathrm{~g}$ and were 11.39 , 8.55 and $9.86 \mathrm{~nm}$ respectively.

The nitrogen adsorption/desorption isotherms (Fig. 2) of the synthesized ordered mesoporous carbons showed a IVtype adsorption isotherms with the capillary condensation at the relative pressure of 0.5-0.8 and H1-type hysteresis loop, indicating the synthesized samples had a uniform mesoporous structure. The narrow pore size distribution (Fig. 3) concentrated at about $3 \mathrm{~nm}$. With the increase of the amount of oxalic acid catalyst, BET specific surface area and the pore volume showed a decreasing trend, but the mean pore diameter decreased at first and then slightly increased [the mean pore diameter was the smallest $(2.84 \mathrm{~nm})$ when the amount of catalyst was $0.1 \mathrm{~g}]$. This indicated the specific surface of the synthesized ordered mesoporous carbons and the pore volume decreased with the increase of the amount of oxalic acid catalyst,

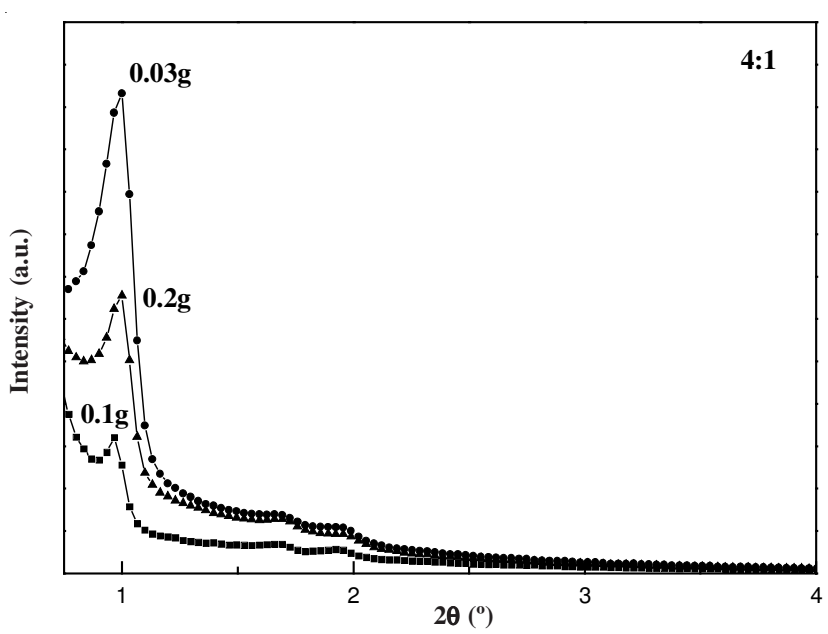

Fig. 1. XRD patterns for mesoporous carbons synthesized with different amount of oxalic catalyst

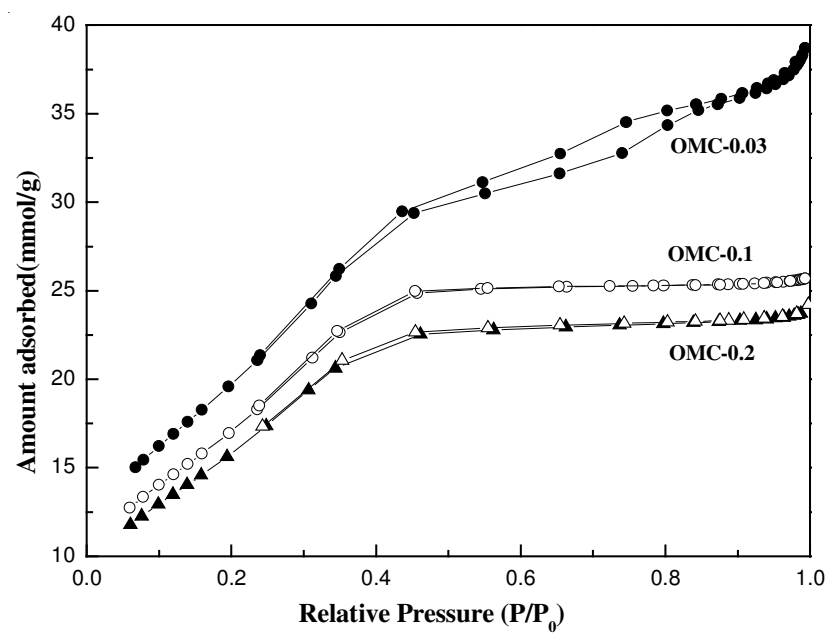

Fig. 2. Nitrogen adsorption/desorption isotherms of the synthesized ordered mesoporous carbons (OMCs)

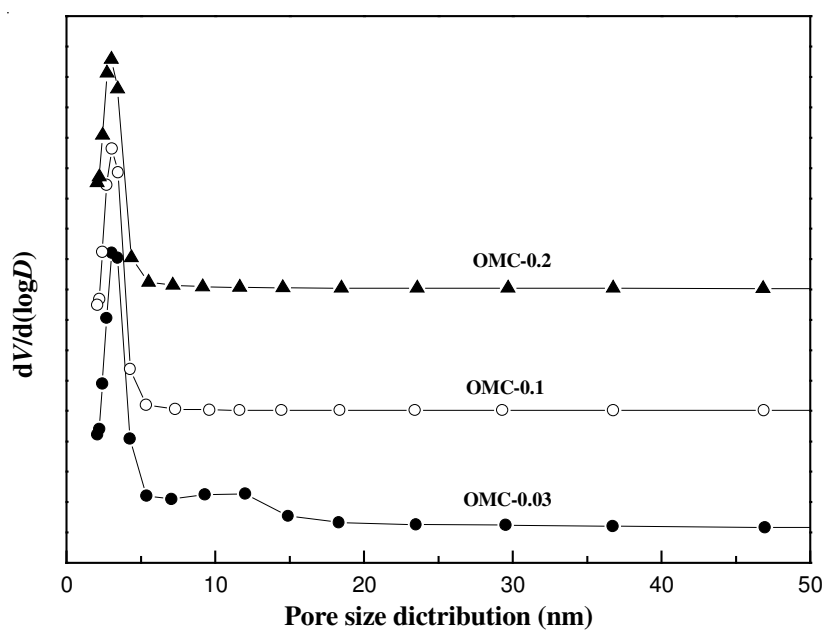

Fig. 3. Pore size distributions of the synthesized ordered mesoporous carbons (OMCs)

TABLE-1

STRUCTURAL PARAMETERS FOR THE SYNTHESIZED ORDERED MESOPOROUS CARBONS (OMCs)

\begin{tabular}{|c|c|c|c|c|c|c|}
\hline Sample & $\mathrm{S}_{\mathrm{BET}}\left(\mathrm{m}^{2} \mathrm{~g}^{-1}\right)$ & $V_{t}\left(\mathrm{~cm}^{3} \mathrm{~g}^{-1}\right)$ & $\mathrm{D}_{\text {pore }}(\mathrm{nm})$ & $\alpha(\mathrm{nm})$ & d-spacing (nm) & Wall thickness (nm) \\
\hline OMC-0.03 & 1640 & 1.34 & 3.61 & 10.89 & 9.43 & 7.28 \\
\hline OMC-0.10 & 1418 & 0.89 & 2.84 & 11.39 & 9.86 & 8.55 \\
\hline $\mathrm{O} 0 \mathrm{C}-0.20$ & 1324 & 0.86 & 2.92 & 11.36 & 9.84 & 8.44 \\
\hline
\end{tabular}




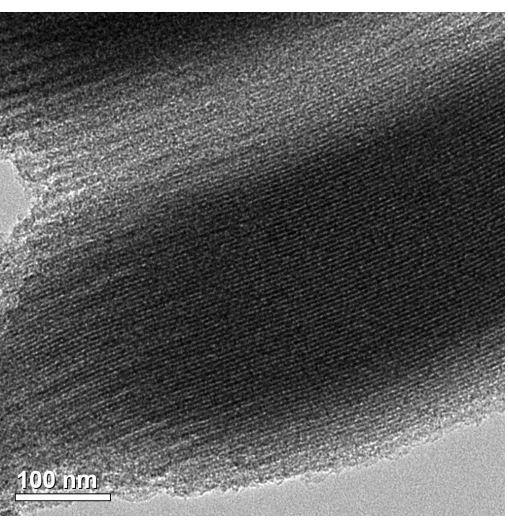

(a) OMC-0.03

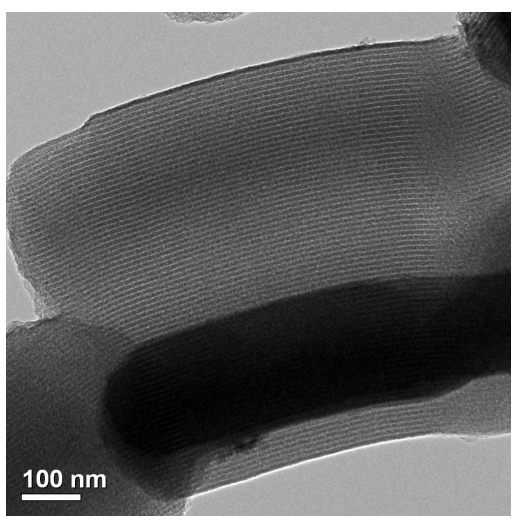

(b) OMC-0.1

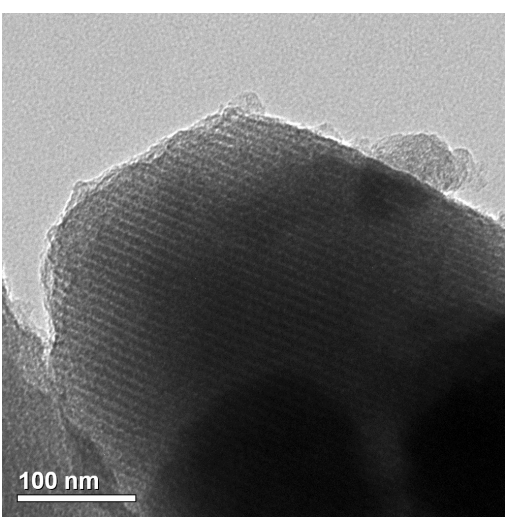

(c) OMC-0.2

Fig. 4. TEM images of the synthesized ordered mesoporous carbons (OMCs)

which may produce the increasing poly-furfuryl alcohol with a higher polymerization degree with the increase of the amount of oxalic acid catalyst so that the cross linking between furfuryl alcohols and the linking points between poly-furfuryl alcohol and silica template increased, producing the higher compression force and lead to a greater structural shrinkage.

The hexagonal structure could be clearly seen from TEM images (Fig. 4) of ordered mesoporous carbons synthesized, confirming the existence of the order, which was consistent with the results from XRD and $\mathrm{N}_{2}$ adsorption test. The pore diameters estimated roughly were $3.8 \mathrm{~nm}$ (OMC-0.03), 2.9 $\mathrm{nm}$ (OMC-0.1) and $3.0 \mathrm{~nm}$ (OMC-0.2).

Fig. 5 showed the Raman spectra of ordered mesoporous carbons synthesized, with two broad peaks observed at wave number of 1314 and $1585 \mathrm{~cm}^{-1}$ corresponding to D-band and $\mathrm{G}$-band respectively. The vibration at $1585 \mathrm{~cm}^{-1}$ ( $\mathrm{G}$ band) due to the interplane $s p^{2} \mathrm{C}-\mathrm{C}$ stretching was the characteristic feature of crystallinity graphite carbon. Another band at around $1314 \mathrm{~cm}^{-1}$ (D band) corresponding to the defects and disorders with regard to the ideal graphitic materials could also be observed. The results indicated that the carbon materials are composed of graphites carbon and amorphous carbon.

The adsorption equilibrium of resorcinol onto the ordered mesoporous carbons was shown in Fig. 6. Langmuir-Freundlich adsorption isotherm equation ${ }^{14}$ was used to fit the adsorption data.

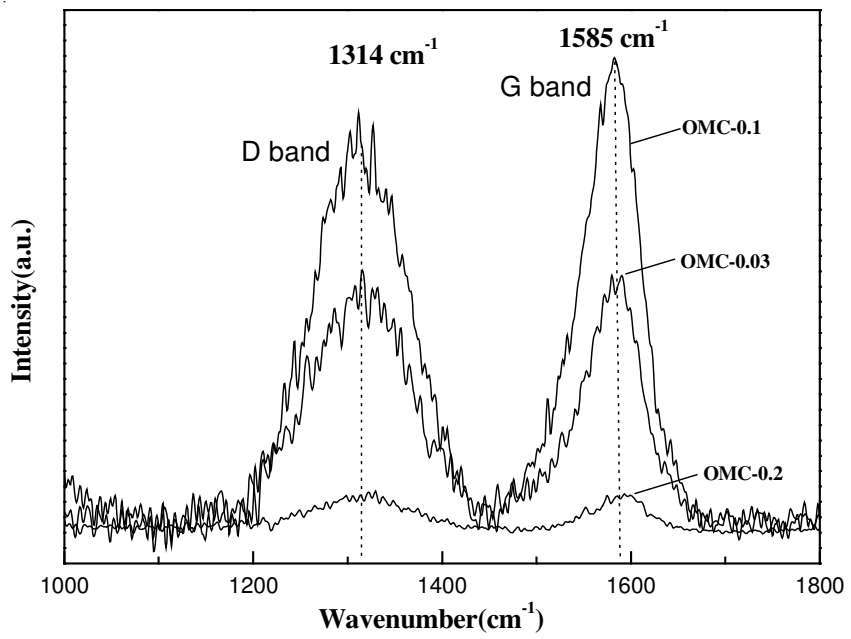

Fig. 5. Raman spectra of the synthesized ordered mesoporous carbons

$$
\mathrm{q}=\mathrm{q}_{\mathrm{m}} \frac{(\mathrm{Kc})^{\mathrm{m}}}{1+(\mathrm{Kc})^{\mathrm{m}}}
$$

where, $\mathrm{q}$ was the adsorption amount, $\mathrm{q}_{\mathrm{m}}$ was the adsorption capacity, $\mathrm{K}$ was the equilibrium constant, c was the concentration of resorcinol at equilibrium and $\mathrm{m}$ was the heterogeneity coefficient. The correlation coefficients $\left(\mathrm{R}^{2}\right)$ were greater than 0.98 . The resorcinol molecules $(0.57 \mathrm{~nm})$ could fit inside the mesopores with large pore diameters $(2.92-3.61 \mathrm{~nm})$.

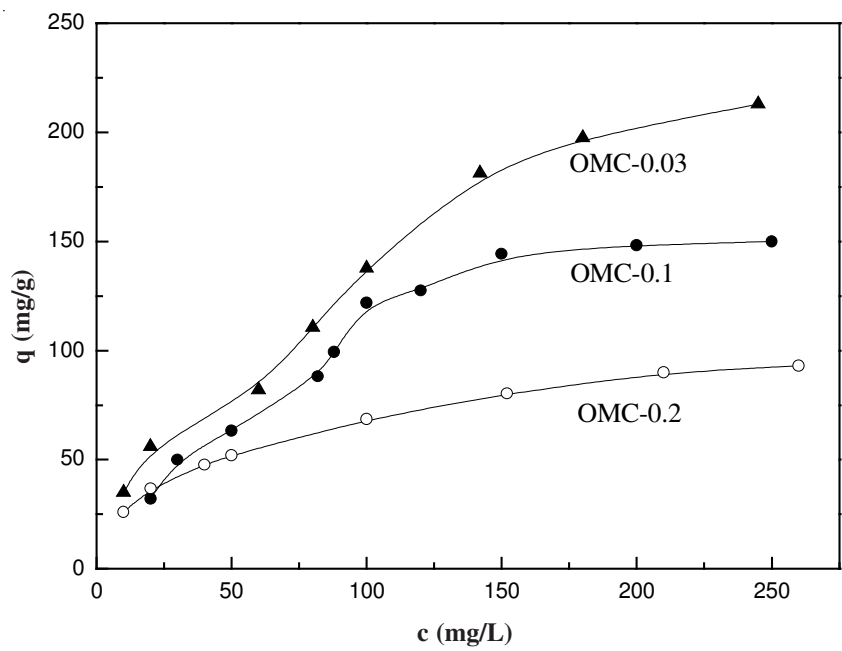

Fig. 6. Adsorption equilibrium of resorcinol onto the obtained ordered mesoporous carbons

The adsorption capacity increased with the increase of the specific surface area and pore volume of ordered mesoporous carbons. For OMC-0.03, qm was $219 \mathrm{mg} / \mathrm{g}$, whereas 152 and $103 \mathrm{mg} / \mathrm{g}$ could be obtained for OMC-0.2 and OMC-0.1, respectively. It was well known that the bigger the specific surface area and pore volume, the more chance and space for resorcinol adsorption on ordered mesoporous carbons were, thus leading to the higher adsorption capacity.

\section{Conclusion}

With the SBA-15 as a template and furfuryl alcohol for carbon source, a series of ordered mesoporous carbons with highly ordered and concentrated pore diameter distribution, high specific surface area, large pore volume, were synthesized under the catalytic action of oxalic acid. The experimental results showed that the synthesized porous carbons exhibited 
highly ordered structures, concentrated pore size distribution, high specific surface area and large pore volume. The amount of oxalic acid catalyst had greater influence on the porous structures. The main reason was due to the increase of the amount of oxalic acid catalyst resulted in the increasing to produce poly-furfuryl alcohols with a higher polymerization degree so that the cross-linking between furfuryl alcohols and the linking points between poly-furfuryl alcohol and silica template increased, producing the higher compression force to lead to a greater structural shrinkage. The adsorption of resorcinol onto ordered mesoporous carbons followed the Langmuir-Freundlich equation.

\section{ACKNOWLEDGEMENTS}

The authors acknowledged the National Science Foundation of China (50802017) and Guangzhou Scientific and Technological Planned Project (2011J4100016).

\section{REFERENCES}

1. X.F. Wang, P. Liu and Y. Tian, Micropor. Mesopor. Mater, 142, 334 (2011).

2. D.Y. Zhao, J.L. Feng and Q.S. Huo, Science, 279, 548 (1998).

3. X.F. Wang, P. Liu, Y. Tian and L.Q. Zang, Micropor. Mesopor. Mater, 145, 98 (2011).

4. Y. Tian, P. Liu, X.F. Wang and H.S. Lin, Chem. Eng. J., 171, 1263 (2011).

5. Y. Tian, P. Liu, J. Wang, X.F. Wang and H.S. Lin, Mater. Lett., 82, 19 (2012).

6. L.M. Guo, L.X. Zhang and J.L. Shi, Chem. Commun., 40, 6071 (2009).

7. S.S. Huang, Y. Fan and J. Lin, J. Phys. Chem. C., 113, 1775 (2009).

8. W. Xing, S.P. Zhuo, W.J. Si and X. Yuan, Acta Chim. Sin., 67, 761 (2009).

9. R. Ryoo, S.H. Joo and S. Jun, J. Phys. Chem. B, 103, 7743 (1999).

10. A.H. Lu and F. Schuth, Adv. Mater., 18, 1793 (2006).

11. D. Mohan, A. Sarswat, V.K. Singh, M. Alexandre-Franco and C.U. Pittman Jr., Chem. Eng. J., 172, 1111 (2011).

12. V.K. Gupta and Suhas, J. Environ. Manag., 90, 2313 (2009).

13. Y. Tian, X.F. Wang and Y.F. Pan, J. Hazard. Mater, 213-214, 361 (2012).

14. A. Derylo-Marczewska, A.W. Marczewski, Sz. Winter and D. Sternik, Appl. Surf. Sci., 256, 5164 (2010). 